\title{
PTFE Graft as a "Bridge" to Communicating Veins Maturation in the Treatment of an Intrahepatic Cholangiocarcinoma Involving the 3 Hepatic Veins. The Minor-but-Complex Liver Resection
}

\author{
Lucio Urbani ${ }^{1}$, Riccardo Balestri ${ }^{1}$, Francesco Sidoti ${ }^{1}$, Juri Riccardo Bernardini ${ }^{1}$, Francesco Arces ${ }^{1}$, \\ Gabriella Licitra $^{2}$, Chiara Leoni ${ }^{2}$, Francesco Forfori ${ }^{2}$, Piero Colombatto ${ }^{3}$, Piero Boraschi ${ }^{4}$, Maura Castagna ${ }^{5}$, and \\ Piero Buccianti ${ }^{1}$ \\ ${ }^{1}$ General Surgery, Azienda Ospedaliero-Universitaria Pisana, Pisa, Italy; ${ }^{2}$ Anesthesiology, Azienda Ospedaliero- \\ Universitaria Pisana, Pisa, Italy; ${ }^{3}$ Hepatology, Azienda Ospedaliero-Universitaria Pisana, Ospedale, Italy; ${ }^{4}$ Radiology, \\ Azienda Ospedaliero-Universitaria Pisana, Pisa, Italy; ${ }^{5}$ Pathology, Azienda Ospedaliero-Universitaria Pisana, Pisa, Italy
}

\begin{abstract}
Background. Parenchyma-sparing liver surgery allows resecting hepatic veins $(\mathrm{HV})$ at the hepatocaval confluence with minor ( $<3$ adjacent segments) liver resections. PTFE graft can be used as a bridge to communicating-veins maturation to ensure the correct outflow of the spared liver. We present a video of an intrahepatic cholangiocarcinoma (IC) involving the three $\mathrm{HV}$ at the hepatocaval confluence treated with this approach.

Methods. In a 50-year old obese (BMI 44.8) male a 6-cm IC involving the hepatocaval confluence was identified during the follow-up for a kidney malignancy. At the preoperative $\mathrm{CT}$ scan the left $\mathrm{HV}$ was not detectable, the middle $\mathrm{HV}$ was incorporated within the tumor, and right HV had a $3-\mathrm{cm}$ contact with the tumor. No communicating veins were evident at preoperative imaging.

Results. After a J-shape thoracophrenolaparotomy, the resection of segments II-III-IVa was partially extended to
\end{abstract}

Electronic supplementary material The online version of this article (doi:10.1245/s10434-016-5526-3) contains supplementary material, which is available to authorized users.

(C) Society of Surgical Oncology 2016

First Received: 28 May 2016;

Published Online: 11 October 2016

L. Urbani

e-mail: 1.urbani@ao-pisa.toscana.it segment VIII-VII and I. The right HV was detached from the tumor, and the middle $\mathrm{HV}$ was reconstructed with a 7-mm ringed-armed PTFE graft anastomosed to V8. Surgery lasted $20 \mathrm{~h}$ and $55 \mathrm{~min}$ with an estimated blood loss of $3500 \mathrm{ml}$, but the postoperative course was uneventful and the patient was discharged on the 14th postoperative day. One month later the CT scan showed a patent PTFE graft with the maturation of communicating-veins. One year later a complete thrombosis of the PTFE graft was observed with normal liver perfusion and function, and the patient was disease-free.

Conclusions. PTFE-based parenchyma-sparing liver resection is a new tool to treat tumors located at the hepatocaval confluence exploiting the maturation of intrahepatic communicating-veins between main HV. 